\title{
Research on the optimal regulation of barrel tea rolling machine based on the tea rolling modeling
}

\author{
Tang Heping ${ }^{1}$ ， Xu Haiwei ${ }^{1}$, Cao Jiangping ${ }^{1}$, Li Gang ${ }^{1}$, Yang Zhuang ${ }^{1}$ ， Zhao Xin ${ }^{2, a}$, \\ $\mathrm{Xie} \mathrm{Chi}^{2, \mathrm{~b} *}$
}

(1. Sichuan Measuring Instrument Technology Company Sichuan, Chengdu, 610021

2. Sichuan university, Sichuan, Chengdu, 610065)

a1548022998@qq.com, ${ }^{b}$ xiechiww@163.com

\begin{abstract}
Keywords: tea rolling, rolling pressure, force model, optimal regulation
Abstract. The tea physical properties and quality are often influenced by the rolling pressure in the tea production. In this paper, the tea rolling modeling in the barrel rolling machine is established by analyzing the force situation of the tea leaves in the barrel rolling machine. The joint rolling curve synthesis of the pressure and rolling disk speed are formulated as a convex optimization form that can be efficiently solved by existing numerical methods. The rolling process automatic control technology is used to control the pressure and the speed parameters in real time. To make the curly tea leaves, the values of tea crowded force are properly changed by measured the values of the barrel internal pressure and the rolling disk speed. The test results show that: the barrel tea rolling machine which has the adjustable speed limit is $60 \mathrm{rads} / \mathrm{min}$; the adjustable pressure limit is $800 \mathrm{~N}$ and the barrel diameter is $550 \mathrm{~mm}$, when the rolling tea weight is between the $500 \mathrm{~g}$ to $4000 \mathrm{~g}$, its the tea breakage rate is less than $10 \%$.
\end{abstract}

\section{Introduction}

In recent years, needle-shaped famous green tea was deeply loved by the masses of tea drinkers because of its beautiful appearance elegant, a feature of tight and thin strip round straight like pine needles and green, long high fragrant, looked green bright and complete and green leaves. In the process of tea processing technology, the purpose of tea rolling process is the destruction of the tea cells effectively, knead a tea juice, which adhere on the leaves and make for brewing, making the leaves beautiful appearance and improving the color and flavor of tea. Literature [1,2] shows that fresh leaves broken degree will affect the absorption of oxygen and the speed of oxygen diffusion into the blade in the fermentation of the leaves, thus affecting the chemical properties of polyphenols in the leaves and texture. Therefore, rolling in the machining process of green tea is one of the important processes to make the needle-shaped famous green tea beautiful appearance, and is the key step to improve the infusing resistance of needle-shaped famous green tea.

At present our country generally used barrel type rolling machine in the production of tea area barrel type rolling machine. leaves knead in the barrel under pressure and the friction force, the function of friction make the leaves into elliptical spiral along the master volume, and under the condition of rolling machine speed is constant, the pressure is the main factors increasing friction and making the leaves rolled. The adjustment of pressure size is the key of the rolling technology [3]. In the process of rolling, barrel type tea rolling mill rolling pressure is generally operated by the operating personnel when pressurized structure for rotary motion. While the automatic rolling machine takes advantage of integration of digital technology and PLC control technology, realizing the tea rolling process automation, but for the stress distribution of rolling lobe in barrel type rolling machine and the analysis of the relationship between the external pressures is insufficient. Therefore, through analyzing structure of kneading disc blade and force of rolling leaf tea in the barrel, establishing the best pressure model, forming the curve associated with rolling plate speed and force parameters, implementing the 
intelligent of the tea rolling processing and satisfying needs for mechanization, intelligent industry in our country tea processing.

\section{Stress Analysis of Tea Rolling Process}

The main frame of tea rolled machine is a parallel rolled three crank linkage mechanisms, which is the installation parameters rolled rib bone plate. When the crank for constant rotary motion, driven rub barrel points in a circular motion, packed in a bucket of rolled leaves rub rubbing plate contact and rub on the disc horizontally in parallel movement, rolled leaves constantly flip, rub, rolled, rolled to achieve the purpose. Rolled rolling machine function is determined by a continuous spiral kneading blade rolled plate inside the barrel has a special shape of the curve as the spiral movement at work to achieve. Rolled plate structure model is shown in the Fig.1.

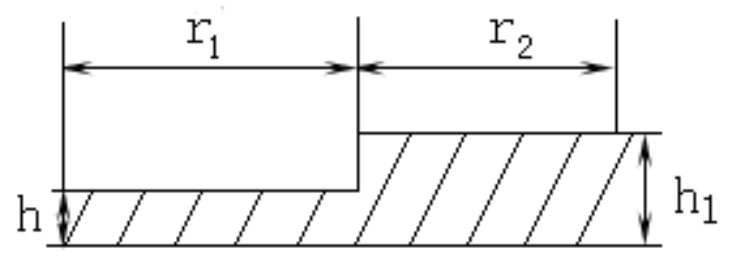

Fig.1 profile graph of rolling disk

In the Fig. 1, r1 and $\mathrm{r} 2$ are respectively the rolled plate and outer radius. Rolled leaves are rolled by the thrust bucket rolled plate, an infinite length assumption is adopted for inside and outside of the rolling bearing disk, and the sector can be rolled rolling disc leaf ladder approximately rectangular ladder. $\mathrm{H}$ is the rolling disc thickness for rolling tooth thickness. Consider rolling disc leaf fan angle, then rolled leaves at any point $r$ suffered by centrifugal force balance conditions,

$$
d m(r+d r) \omega^{2}-\tau(r+d r) d \varphi d r=0
$$

In the formula (1), $\tau$ is the centrifugal force balance tangential friction, $m$ is the mass of rolled leaves, $d m=\rho(r+d r) d \varphi d r d h, \quad \rho$ is the rolled leaf density, into the formula (1) and ignore the high order terms,

$$
\frac{d \tau}{d r}=\rho \omega^{2} r
$$

According to Newton's law of friction, and centrifugal force balance is tangential friction:

$$
\tau=-\frac{d v}{d r}
$$

In the formula (3), $v$ is the rolled leaves into the radial velocity, and into the formula (2):

Two times of integration:

$$
\frac{d}{d r}\left(-\frac{d v}{d r}\right)=\rho\left(\frac{\omega}{h} r\right)^{2} r
$$

$$
v=-\rho\left(\frac{\omega}{h^{2}} r^{4}\right) r+c_{1} r+c_{2}
$$

Rolling disc center relative velocity is considered to be zero, the velocity boundary of $h_{1}$ is $v$, on behalf of the boundary conditions is considered:

$$
c_{2}=0, c_{1}=\rho \omega^{2}\left(h_{1}-h\right)
$$

Thus rolled leaves produce radial centrifugal force can be obtained under the flow,

$$
Q=\int_{0}^{r} 2 \pi v r d r=\rho \pi \omega^{2} r^{2}\left(h_{1}-h\right)^{3}
$$

Leaves rolled by centrifugal force is considered with the disc radius, and can be obtained formula (8) 


$$
d p=Q \cdot \frac{d r}{r}
$$

Rolled leaves to withstand centrifugal force can be obtained:

$$
\begin{aligned}
F_{1} & =-\iint d p d A \\
& =-\iint \rho \omega^{2} r d r \pi r d r \\
& =-\rho \pi \omega^{2} \int_{r_{1}}^{r_{2}} r^{3} d r \\
& =-\rho \pi \omega^{2}\left(r_{2}^{4}-r_{1}^{4}\right)
\end{aligned}
$$

The pressure generate by the pressure supply system of the rolled leaves:

$$
\begin{aligned}
F_{2} & =\int_{r_{1}}^{r_{2}} 2 \pi \cdot r p(r) d r \\
& =2 \pi P\left(r_{2}^{2}-r_{1}^{2}\right)
\end{aligned}
$$

$\mathrm{P}$ is for pressure system provides dynamic pressure in the tea rolling process. The rolled barrel suffered dynamic force is $F=F_{1}+F_{2}$ that obtained from the above rolled leaves. Under the certain conditions of rolled plate size, tea by the rolling force, pressure supply system pressure and kneading disk rotation speed provided there is a direct link.

\section{Optimization Design of Rolling Machine}

Tea-grinding is performed under a certain pressure provided by pressure supply system, moreover sufficient grinding pressure not only ensures that the real contact area between the grinder and tea but also a certain degree of process efficiency. Analysis of barrel-rolling machine using cylindrical coordinates ,considering the polarization angle of cylindrical coordinates is, of which is the angle of inclination and is the angle of the ellipse, as shown in Figure 2.

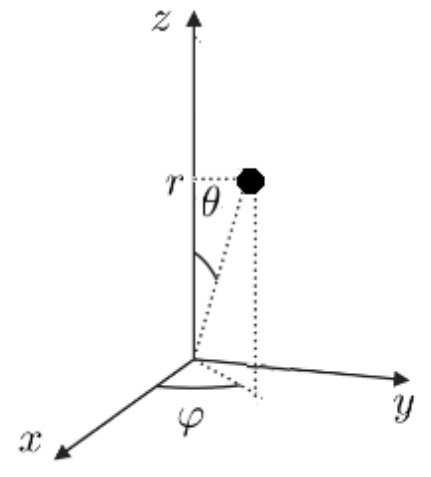

Fig. 2 the unite tea coordinate in barrel rolling machine

So pressure supply system provides the pressure can be expressed as:

$$
\mathbf{P}=\left[\begin{array}{c}
P_{H} \\
P_{v}
\end{array}\right]=\| \mathbf{P}\left[\begin{array}{cc}
\cos \theta & -\sin \theta \\
\sin \theta & \cos \theta
\end{array}\right]\left[\begin{array}{c}
\cos \phi \\
j \sin \phi
\end{array}\right]
$$

There $P_{H}$ and $P_{v}$ stand for the horizontal and vertical components respectively, $\|\mathbf{P}\|$ for the pressure amplitude. An ideal optimization control designed should be met:

$$
P_{v}(\theta, \phi) / P_{H}(\theta, \phi)=\|\mathbf{P}\| e^{j \tan \left(\frac{h_{1}}{r_{1}+r_{2}}-\frac{h}{r_{1}}\right)} \quad \omega_{\min } \leq \omega \leq \omega_{\max }
$$

The $e^{j \tan \left(\frac{r_{2}}{r_{1}+r_{2}}\right)}$ is the phase difference between the rolling plates. 


$$
\left|P_{\nu}(\theta, \phi)-\|\mathbf{P}\| e^{j \tan \left(\frac{h_{1}}{r_{1}+r_{2}}-\frac{h}{r_{1}}\right)} P_{H}(\theta, \phi)\right| \geq P_{\text {min }}
$$

That $P_{\min }$ is the minimum pressure provided by pressure supply system. According to Cauchy's in equality we can obtain:

$$
\left|P_{v}(\theta, \phi)\right| /\left|P_{H}(\theta, \phi)\right| \geq|| \mathbf{P} \| e^{j \tan \left(\frac{h_{1}}{r_{1}+r_{2}}-\frac{h}{r_{1}}\right)}\left|+P_{\text {max }} /\right| P_{H}(\theta, \phi) \mid
$$

Maximum constraints pressure can be expressed as:

$$
\left(\left|P_{H}(\theta, \phi)\right|^{2}+\left|P_{v}(\theta, \phi)\right|^{2}\right)^{1 / 2} \leq P_{\max } \quad \omega_{\min } \leq \omega \leq \omega_{\max }
$$

That $P_{\max }$ is the maximum pressure provided by tea pressure supply system. In equation (13), if $P_{\min }$ is small, $P_{V}(\theta, \phi)$ is approximately equal to $\|\mathbf{P}\| e^{j \tan \left(\frac{h_{1}}{r_{1}+r_{2}}-\frac{h}{r_{1}}\right)} P_{H}(\theta, \phi)$, we can get:

$$
\frac{\partial P_{V}(\theta, \phi)}{\partial \omega} \approx\|\mathbf{P}\| e^{j \tan \left(\frac{h_{1}}{r_{1}+r_{2}}-\frac{h}{r_{1}}\right)} \frac{\partial P_{H}(\theta, \phi)}{\partial \omega}
$$

You can see from above, if at a certain speed, pressure of tea rolling barrels in the direction of $h$ polarization and $\mathrm{v}$ polarization coordinates will be bound. Therefore, the pressure and rate objective function of pressure supply equipment for tea can be expressed as:

$$
\max _{\omega}\left|\frac{\partial P(\theta, \phi)}{\partial \omega}\right|^{2}
$$

Above can be viewed as optimization problems for solving the following objective function and constraints:

$$
\begin{array}{ll}
\max _{\omega} & R_{e}\left(\frac{\partial P(\theta, \phi)}{\partial \omega}\right) \\
\text { s.t. } & \operatorname{Im}\left(\frac{\partial P(\theta, \phi)}{\partial \omega}\right)=0 \\
& P_{H}(\theta, \phi)=0 \quad P_{V}(\theta, \phi)=0, \quad \text { s.t. } \quad \omega=0 \\
& \left(\left|P_{H}(\theta, \phi)\right|^{2}+\left|P_{v}(\theta, \phi)\right|^{2}\right)^{1 / 2} \leq P_{\max } \quad \omega_{\min } \leq \omega \leq \omega_{\max } \\
& \left|P_{v}(\theta, \phi)-\right| \mathbf{P}\left|e^{j \tan \frac{h_{1}}{h_{1}+r_{2}} \frac{h}{r_{1}}} P_{H}(\theta, \phi)\right| \geq P_{\min } \quad \omega_{\min } \leq \omega \leq \omega_{\max }
\end{array}
$$

This optimization problem is a convex optimization problem, which can use a numerical method [4] or use MATLAB CVX[5]optimization toolbox to solve.

\section{Intelligent Barrel Rolling Machine Design Simulation}

Intelligent barrel rolling machine is mainly composed of rolling barrel, rolling plate, pressure sensors, hydraulic control structures. The reduction gear mechanism of control box is made up of a 200W single-phase ac motor and a gear reducer, driven by a pulley. The pressure sensing structure has a dynamic real-time monitoring for rolled leaves pressure in the rolling process. In order to reduce the mechanical wear and tear and energy consumption of the rolling machines, pressure real-time adjusting effect is achieved by the real-time action of pressure perform element, rolling force of rolling leaf is controlled through the closed-loop control system. The result of statistics is shown in Table 1. 
Tab. 1 Statistics of tea breakage rate

\begin{tabular}{|c|c|c|c|c|c|c|c|}
\hline Method & Total (g) & Break (g) & Rate (\%) & Method & Total (g) & Break (g) & Rate (\%) \\
\hline \multirow{10}{*}{ Manual } & 250 & 26 & $10.2 \%$ & \multirow{10}{*}{ Machine } & 250 & 36 & $14.4 \%$ \\
\hline & 500 & 82 & $16.4 \%$ & & 500 & 42 & $8.4 \%$ \\
\hline & 1000 & 137 & $13.7 \%$ & & 1000 & 67 & $6.7 \%$ \\
\hline & 1500 & 192 & $12.8 \%$ & & 1500 & 97 & $6.5 \%$ \\
\hline & 2000 & 262 & $13.1 \%$ & & 2000 & 130 & $5.2 \%$ \\
\hline & 2500 & 328 & $13.1 \%$ & & 2500 & 150 & $4.5 \%$ \\
\hline & 3000 & 426 & $14.2 \%$ & & 3000 & 135 & $5.5 \%$ \\
\hline & 3500 & 647 & $18.5 \%$ & & 3500 & 213 & $6.1 \%$ \\
\hline & 4000 & 940 & $23.5 \%$ & & 4000 & 288 & $7.2 \%$ \\
\hline & 4500 & 1548 & $34.4 \%$ & & 4500 & 459 & $10.2 \%$ \\
\hline
\end{tabular}

Through the Table 1 data, when the weight of rolling tea leaves is light, the result of manual method is better than the machine. For the artificial method of rolling tea, skilled master is often difficult to secure the damage rate less than $10 \%$ of the tea. Through the design of the rolling machine in rolling leaf for $2000 \mathrm{~g}$ to $3000 \mathrm{~g}$ realize the best state of rolling fitting for rolling speed and the hydraulic system for disc pressure curve. Under pressure control mode, high speed low rolling way is adopted when rolling leaf weight is lighter, high speed low pressure after high pressure at low speed rolling method is adopted when rolling leaf weight heavier. Let the pressure and rolling speed has certain elasticity change space, so that the pressure on rolling leaf neither mutation or uniform, so can not only ensure the rolling leaf rolling rollover effect also can reduce the wastage of the rolling machine. Table 1 shows that under the condition of the tea breakage rate of less than $10 \%$, the best rolling weight of rolling tea is from $500 \mathrm{~g}$ to 4000 .

\section{Summary}

Based on the stress distribution of tea in the rolling machine barrel, set up the force model of tea in the rolling process is set up. Rolling speed and the hydraulic system for disc pressure curve is obtained by optimization toolbox for solving the convex optimization problem. Real-time monitoring barrel rolling machine rolling pressure values, and appropriately changing barrels of internal pressure and rolling plate speed according to how much leaf placed, effectively regulate squeeze intensity of tea to achieve the goal of curly tea and the "first re-light, gradually pressing the last pine pressure" characteristics of artificial rub tea. In the rolling barrel, revolutions cap can be adjusted to $60 \mathrm{rads} / \mathrm{min}$, the pressure cap can be adjusted to $800 \mathrm{n}$, when the knead barrel diameter is $550 \mathrm{~mm}$, the best rolling tea weight of the rolling machine is from $500 \mathrm{~g}$ to $4000 \mathrm{~g}$, tea damage rate is less than $10 \%$.

\section{References}

[1] J. van der Geer, J.A.J. Hanraads, R.A. Lupton, The art of writing a scientific article, J. Sci. Commun. 163 (2000) 51-59.

[2] W. Strunk Jr., E.B. White, The Elements of Style, third ed., Macmillan, New York, 1979.

[3] G.R. Mettam, L.B. Adams, How to prepare an electronic version of your article, in: B.S. Jones, R.Z. Smith (Eds.), Introduction to the Electronic Age, E-Publishing Inc., New York, 1999, pp. 281-304.

[4] R.J. Ong, J.T. Dawley and P.G. Clem: submitted to Journal of Materials Research (2003)

[5] P.G. Clem, M. Rodriguez, J.A. Voigt and C.S. Ashley, U.S. Patent 6,231,666. (2001)

[6] Information on http://www.weld.labs.gov.cn 